\title{
RESEARCH HIGHLIGHT Seeing is believing: a novel tool for quantitating mitophagy
}

\author{
Liming Wang ${ }^{1}$ and Han-Ming Shen ${ }^{2}$ \\ Cell Research (2020) 30:715-716; https://doi.org/10.1038/s41422-020-0360-3
}

\begin{abstract}
As dysfunctional mitophagy is linked to multiple human diseases, there is an urgent and important unmet need for developing reliable, reproducible, and quantitative tools to study the mechanisms and biological functions of mitophagy. Recently, Katayama and colleagues developed one such tool, named mito-SRAl, which makes it possible to precisely interpret mitophagy in living and fixed specimens.
\end{abstract}

Mitophagy is a process that specifically degrades damaged or superfluous mitochondria via the autophagy-lysosome machinery. ${ }^{1,2}$ As a main mitochondrial quality control, dysfunctional mitophagy, mostly defective mitophagy, is associated with various human diseases, including neurodegenerative disorders, cancer, cardiovascular disease, autoimmune disease, and aging. Thus, in the past decade, mechanistic and functional studies of mitophagy have attracted extensive attention. Among many important mitophagy regulators identified so far, PINK1 and Parkin are the key factors to orchestrate the mitophagic process. First, upon mitochondrial damage, PINK1 as a serine/threonine kinase phosphorylates ubiquitin (Ub) and Parkin, forming the first feedforward amplification loop to initiate mitophagy. Second, TBK1 phosphorylates autophagy receptors, such as OPTN and NDP52, forming the second feedforward amplification loop to mediate the specific recognition of damaged mitochondria by autophagy machinery. Third, ULK1 complex recruits LC3 and more autophagy receptors onto damaged mitochondria, forming the third feedforward amplification loop to sequester damaged mitochondria with autophagosomes. Finally, autophagosomes containing damaged mitochondria are delivered to lysosome for degradation. Mutation or deletion of PINK1 and Parkin are found in autosomal-recessive juvenile Parkinsonism (AR-JP), ${ }^{3}$ which theoretically results in defective/insufficient mitophagy and dopaminergic neuron death. Therefore, it is believed that enhancement of mitophagy by pharmacological agents may provide a therapeutic opportunity for some neurodegenerative disorders such as Parkinson's disease (PD).

One important limitation over the years is the lack of a reliable and quantitative tool for measuring mitophagy, especially in in vivo animal studies. At present, mt-Keima and mito-QC are two widely used tools to analyze mitophagy in cultured cells and in animal models. ${ }^{4,5}$ The readout of both tools depends on the ratio of two differently colored fluorescent (green vs. red) intensities owing to the different acid environments between mitochondria and lysosome. In brief, mt-Keima (Keima protein fused with mitochondrial matrix-targeting sequence (MMTS)) displays green fluorescence in healthy mitochondria $(\mathrm{pH} \sim 8.0)$ with the shorter excitation $440 \mathrm{~nm}$ wavelength, whereas $\mathrm{mt}$-Keima displays red fluorescence in lysosome $(\mathrm{pH} \sim 4.5)$ with the longer excitation $586 \mathrm{~nm}$ wavelength; thus, mt-Keima can be used as an indicator for mitolysosomes. ${ }^{6}$ However, these properties of mt-Keima are highly dependent on the lysosomal acidic environment, which makes it not applicable for cell and tissue samples with fixation. On the other hand, mito-QC is a mCherry-GFP tandem reporter fused with the mitochondrial-targeting sequence (MTS) from FIS1 that drives localization of this reporter on outer mitochondrial membrane (OMM). ${ }^{4}$ When the mito-QC reporter associated with the damaged mitochondria is delivered to lysosomes, the GFP fluorescence is irreversibly quenched, whereas the mCherry fluorescence resistant to lysosomal acidic environment represents the degree of mitophagic flux. Thus, mito-QC reporter can be used for fixed samples. Nevertheless, it is challenged by the facts that most of OMM proteins including FIS1 are degraded by proteasome upon mitochondrial damage. ${ }^{7}$ Another concern is that FIS1 can localize in other organelles such as peroxisomes especially under the constitutive overexpression condition. To overcome these drawbacks, novel tools with accurate mitophagic readout are needed.

A recent study published by Katayama and colleagues in Cell introduced a novel tool, named mito-SRAl, to quantitatively study mitophagy in living and fixed specimens. ${ }^{8}$ They screened the library from Anthozoans and found one special fluorescent protein (afCFP, acid-fast CFP). AfCFP is able to emit full fluorescence at $\mathrm{pH}$ from 4 to 8 with the same spectral properties, and is resistant to lysosomal proteolysis (they renamed afCFP as TOLLES). Based on these unique properties, they generated the mito-SRAI reporter through a tandem fusion of TOLLES and YPet with a MMTS. YPet is an irreversibly acid-denatured yellow-emitting protein, which can be degraded by pepsin. To increase the mitochondrial localization of this reporter, Katayama et al. modified the C-terminus of mtSRAI to reduce cytosolic distribution. Thus, mito-SRAI possesses the following characteristics: (1) the opposite extremes of TOLLES and YPet in lysosomal acidic environment; (2) the quantifiable efficiency of fluorescence resonance energy transfer in the tandem construct; (3) complete mitochondrial localization; and (4) resistant to proteasomal degradation.

Using this tool, they first confirmed that mito-SRAI provided reliable and quantifiable mitophagic readout in living and fixed cultured cells after the treatment of mitochondrial damaging agents (CCCP and FCCP), by either fluorescence microscopy or flow cytometry. Next, the authors utilized this mitophagy reporter and performed an image-based screening to identify novel Parkindependent mitophagy inducers. To achieve the therapeutic goals, the mitophagy inducers should meet the following requirements: (1) do not affect normal mitochondrial function, such as mitochondrial membrane potential and ATP production; (2) only remove damaged mitochondria but not healthy mitochondria; and (3) have known molecular targets. Among 76,000 compounds

\footnotetext{
${ }^{1}$ Department of Physiology, Yong Loo Lin School of Medicine, National University of Singapore, Singapore, Singapore and ${ }^{2}$ Faculty of Health Sciences, University of Macau, Macau, China

Correspondence: Liming Wang (phswl@nus.edu.sg) or Han-Ming Shen (hmshen@um.edu.mo)
}

Published online: 19 June 2020 


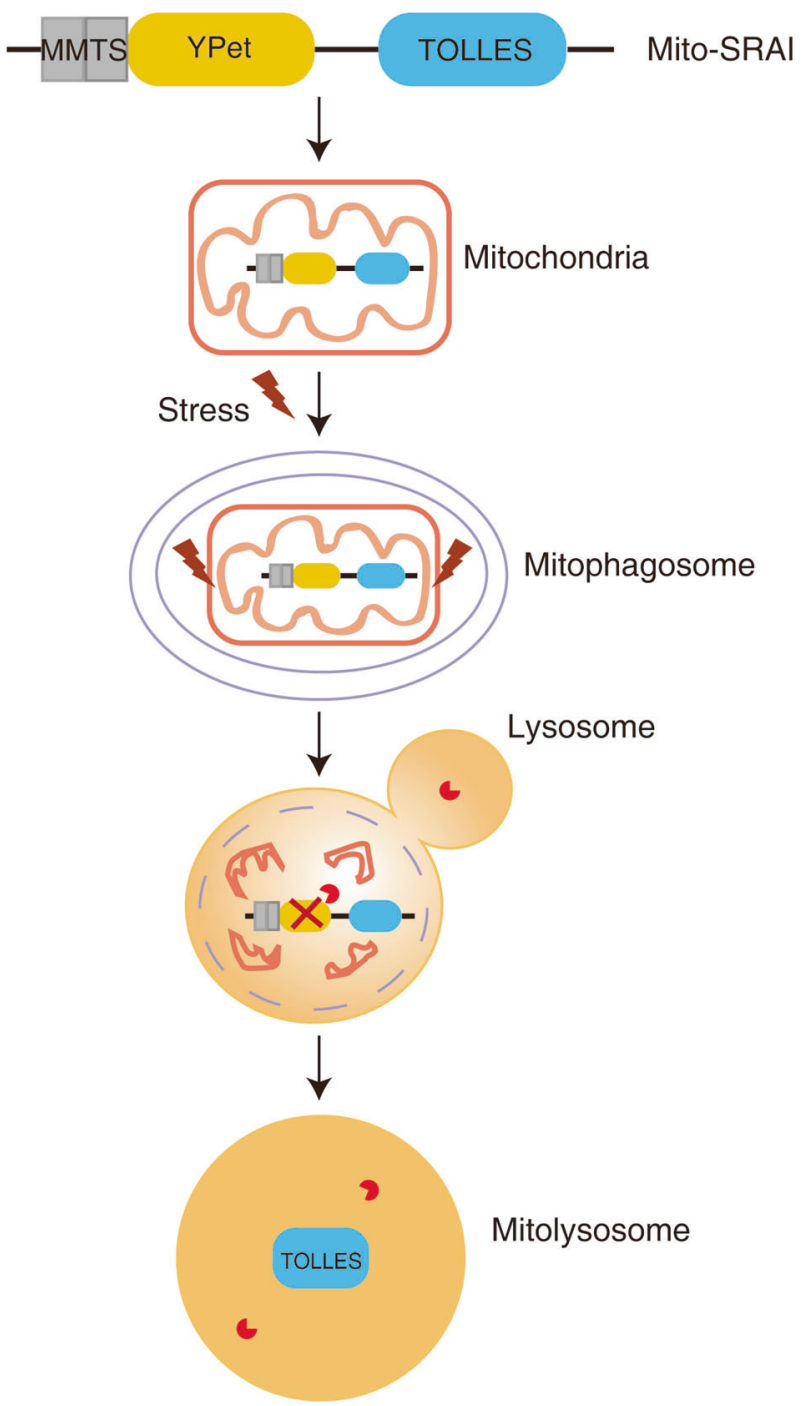

Fig. 1 Illustration of how to use mito-SRAl to analyze mitophagic flux. Mito-SRAl is a tandem construct containing a MMTS and two fluorescent protein tags, YPet and TOLLES. When mitochondria are healthy, mito-SRAI emits both yellow and cyan fluorescence in mitochondria matrix $(\mathrm{pH} \sim 8)$. When mitochondria are damaged, mitochondria are engulfed by autophagosomes and delivered to lysosomes to form mitolysosomes. Within the acidic environment of mitolysosomes ( $\mathrm{pH} \sim 4.5$ ), the YPet (yellow fluorescence) is irreversibly quenched and/or digested, whereas TOLLES remains completely intact and emits full cyan fluorescence. Thus, the ratio (TOLLES vs. YPet) can be used as an index of mitophagic activity. screened, only one compound named T-271 meets the above criteria and appears to be promising for potential clinical trials. In addition, Katayama et al. generated an in vivo mouse model by expressing mito-SRAI in neurons, and found that dopaminergic neurons rather than other neurons could not execute mitophagy after neurotoxin (6-OHDA) treatment, leading to lesions and neurodegeneration. Such findings thus directly support the notion that mitophagy has vital roles in neurodegenerative disorders. Using this tool, many of the previously reported positive or negative regulators of mitophagy could be tested quantitatively under both in vitro cell culture and in vivo animal models. For instance, it is possible that restoration of mitophagy in dopaminergic neuron by activating positive regulators such as PINK1 and Parkin, and/or inactivating negative regulators such as USP30 and PTEN-L, will provide therapeutic avenues towards PD. ${ }^{9,10}$

Overall, Katayama et al. developed a powerful mitophagic reporter, mito-SRAI (Fig. 1), which can provide reliable, reproducible, and quantitative readouts of mitophagic flux. It remains to be tested for the efficacy of mito-SRAl to analyze mitophagy in other animal models such as Drosophila and Caenorhabditis elegans. It would be also interesting to further examine whether mito-SRAI is suitable for other mitophagy pathways, such as BNIP3/NIX or FUNDC1 receptor-mediated mitophagy pathway, or other stresses, such as hypoxia.

\section{REFERENCES}

1. Wang, L., Qi, H., Tang, Y. \& Shen, H. M. Trends Biochem. Sci. 45, 58-75 (2020).

2. Lou, G. et al. Trends Mol. Med. 26, 8-20 (2020).

3. Pickrell, A. M. \& Youle, R. J. Neuron 85, 257-273 (2015).

4. McWilliams, T. G. et al. J. Cell Biol. 214, 333-345 (2016).

5. Sun, N. et al. Nat. Protoc. 12, 1576-1587 (2017).

6. Katayama, H., Kogure, T., Mizushima, N., Yoshimori, T. \& Miyawaki, A. Chem. Biol. 18, 1042-1052 (2011).

7. Chan, N. C. et al. Hum. Mol. Genet. 20, 1726-1737 (2011).

8. Katayama, H. et al. Cell 181, 1176-1187 (2020).

9. Bingol, B. et al. Nature 510, 370-375 (2014).

10. Wang, L. et al. Cell Res. 28, 787-802 (2018). 